Chirurg 2012 $\cdot 83: 7-8$

DOI 10.1007/s00104-011-2145-1

Online publiziert: 18. November 2011

(c) Springer-Verlag 2011

\title{
J. Jähne
}

Zentrum Chirurgie, Klinik für Allgemein- und Visceralchirurgie, Schwerpunkt für endokrine und onkologische Chirurgie, Diakoniekrankenhaus Henriettenstiftung Hannover gGmbH, Hannover

\section{Update Magenchirurgie}

\section{Sind Neuerungen wirklich immer besser?}

Angesichts einer praktisch nicht mehr existenten elektiven Magenchirurgie wegen benigner Erkrankungen einerseits und einer zumindest in Deutschland weitgehend akzeptierten Resektionsstrategie beim Magenkarzinom andererseits stellt sich grundsätzlich die Frage, ob es in der chirurgischen Behandlung von Magenerkrankungen tatsächlich Neuerungen gibt, die ein Leitthemenheft rechtfertigen. Die vorliegenden Beiträge zeigen eindrucksvoll, dass insbesondere die chirurgische Therapie des Magenkarzinoms kontinuierlichen Veränderungen unterliegt, die zum gegenwärtigen Zeitpunkt durchaus Anlass zur kritischen Diskussion geben.

D) Eine unkritische Anwendung
von Neuerungen ist nicht
zum Vorteil der Patienten

Gerade in der Behandlung des Magenfrühkarzinoms waren in der Vergangenheit durch die histologieorientierte Resektion des Primärtumors einschließlich einer systematischen Lymphadenektomie exzellente Langzeitergebnisse erzielbar. Durch die Verbesserung endoskopischer Resektionstechniken und auch in Anerkennung der Tatsache, dass eine Gastrektomie beim Magenfrühkarzinom durchaus eine gewisse Übertherapie sein mag, hat sich gerade das endoskopisch-interventionelle Armentarium zur Behandlung dieser Frühkarzinome substanziell verändert. Die Arbeitsgruppe um Ell, Wiesbaden, gehört hier sicher zu den Pionieren dieser Behandlung, und der Beitrag von Manner et al. unterstreicht die vielfältigen endoskopischen Optionen und besonders auch die exzellenten Langzeitergebnisse.

Es ist durchaus mach- und onkologisch vertretbar, Magenfrühkarzinome endoskopisch kurativ zu therapieren. Dennoch dürfen einige Aspekte dieser Arbeit nicht unkommentiert bleiben. Zum einen handelt es sich um die Erfahrungen aus einem hochspezialisierten Zentrum, sodass die Ergebnisse nicht zwangsläufig auf die Flächenversorgung übertragbar sind. Im Gegenteil - es droht eine unkritische Anwendung, die möglicherweise einer Reihe von Patienten und ihrer Erkrankung nicht gerecht wird. Die Autoren schreiben selbst, dass es darauf ankommt, die richtigen Kandidaten für eine kurative endoskopische Therapie auszuwählen. Hochauflösende Videoendoskopie, Chromoendoskopie und Endosonographie sind unverzichtbare Methoden mit einem entscheidenden Nachteil - sie hängen sehr von der Erfahrung des Untersuchers ab! Aus chirurgisch-onkologischer Sicht sind Piecemeal-Resektionen abzulehnen, zumal sie mit einer relevanten Rezidivrate einhergehen - dennoch sehen die Autoren für diese Resektionsstrategie durchaus eine Möglichkeit zur Entfernung größerer Läsionen. Damit geht dann auch die Frage einher, ob die Resektionskriterien nicht weiter angelegt werden können. So exzellent die Ergebnisse sind, so kritisch sollten diese Aspekte im interdisziplinären Dia$\log$ erörtert werden!

Die endoskopischen Resektionstechniken müssen sich natürlich auch zunehmend mit den Möglichkeiten der minimal-invasiven Magenchirurgie messen 
lassen. Was ist besser - ein endoskopisch R1-reseziertes Magenfrühkarzinom erneut endoskopisch anzugehen oder eine laparoskopische Wedge-Resektion bzw. partielle Magenresektion? Der Beitrag von Ludwig et al. verdeutlicht, dass die Magenchirurgie durch die laparoskopischen Resektionsverfahren eine signifikante Erweiterung der technischen Möglichkeiten erfahren hat. Dies gilt besonders für die laparoskopische Resektion von gastrointestinalen Stromatumoren. Selbst ausgedehnte Resektionen einschließlich einer systematischen Lymphadenektomie sind laparoskopisch durchführbar, setzten allerdings eine hohe Expertise voraus und sind sicher mit einer substanziellen Lernkurve verbunden. Daher sollten beim fortgeschrittenen Magenkarzinom, besonders aber beim Karzinom am gastroösophagealen Übergang, wohl unverändert primär offene Resektionstechniken zum Einsatz kommen.

Gerade bei den letztgenannten Tumoren hat die neue TNM-Klassifikation $\mathrm{zu}$ erheblichen Diskussionen geführt. Schuhmacher et al. unterstreichen, dass es sich zunächst bei der neuen TNM-Klassifikation um eine Neubewertung der Prognoseparameter der Karzinome des gastroösophagealen Übergangs handelt, die eine schlechtere und dem Ösophaguskarzinom vergleichbare Prognose haben. Unmittelbare chirurgisch-technische Konsequenzen ergeben sich daraus nicht. Dennoch steht zu befürchten, dass im klinischen Alltag die fundierte Einteilung der gastroösophagealen Übergangskarzinome nach Siewert in die AEG-Typen IIII mit den entsprechenden Resektionsstrategien (leider) an Bedeutung verliert. In dem Moment, in dem diese Tumoren pauschal als Ösophaguskarzinome klassifiziert werden, ist es nur zu wahrscheinlich, dass diese Karzinome dann auch entsprechend operativ behandelt werden und somit ein etabliertes und exzellentes Klassifikationssystem an Bedeutung verliert. Darüber hinaus sollte es nicht verwundern, wenn die nächstjährigen Krankenhausstatistiken eine deutliche Zunahme der Ösophaguskarzinome zeigen, ohne dass dies de facto der Fall ist. Der Reiz, durch TNM-gestützte Empfehlungen die momentan in der Diskussion befindliche Mindestmengenregelung zu erfüllen und
Mehrerlöse $\mathrm{zu}$ generieren, ist einfach $\mathrm{zu}$ hoch!

In diesem Kontext muss auch berücksichtigt werden, dass die aktuelle S3-Leitlinie zur Therapie des Magenkarzinoms unverändert die AEG-Klassifikation zur Resektion der Karzinome des gastroösophagealen Überganges empfiehlt. Die von Meyer et al. dargestellte Leitlinie sollte fester Bestandteil einer jeden Tumorkonferenz sein! Wie oft erleben wir als Chirurgen, dass von onkologischer Seite nach R0-Resektion aufgrund bestimmter Tumorkonstellationen eine adjuvante Chemotherapie vorgeschlagen wird? Wie häufig wird heute gerade auch bei den technisch gut zu resezierenden uT2-Tumoren eine neoadjuvante Chemotherapie propagiert? Für letztere handelt es sich - wohlbemerkt - um Kann-Empfehlungen, die allerdings immer mehr dazu führen, dass wir uns als Chirurgen rechtfertigen müssen, wenn wir eine primäre Resektion empfehlen. Und letztlich ist auch die postoperative adjuvante Chemotherapie im perioperativen Therapiekonzept kritisch zu hinterfragen, da die prognostische Relevanz derzeit unklar ist. Es ist unsere Aufgabe als Chirurgen, im interdisziplinären Dialog die chirurgischen Standpunkte klar darzulegen und vielleicht auch unsere Patienten vor einer multiprofessionellen Übertherapie zu schützen. In diesem Kontext ist es wichtig, dass wir uns auch auf Gebieten, auf denen vermeintlich alles geklärt ist, immer wieder kritisch mit den Neuerungen auseinandersetzen, denn nicht immer ist das Neue unbedingt besser.

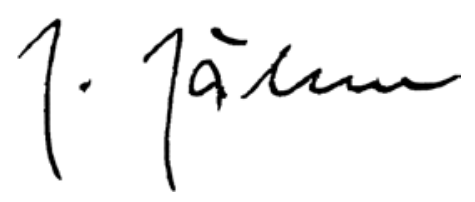

Prof. Dr. J. Jähne

\section{Korrespondenzadresse}

Prof. Dr. J. Jähne

Zentrum Chirurgie, Klinik für Allgemein- und Visceralchirurgie, Schwerpunkt für endokrine und onkologische Chirurgie,

Diakoniekrankenhaus Henriettenstiftung

Hannover gGmbH,

Marienstr. 72-90, 30171 Hannover

joachim.jaehne@ddh-gruppe.de 